\title{
Promouvoir la diversité culturelle (2) ?
}

\section{Béatrice Fleury et Jacques Walter}

\section{(2) OpenEdition}

Journals

Édition électronique

URL : http://journals.openedition.org/questionsdecommunication/877

DOI : 10.4000/questionsdecommunication. 877

ISSN : 2259-8901

\section{Éditeur}

Presses universitaires de Lorraine

\section{Édition imprimée}

Date de publication : 1 décembre 2008

Pagination : 143-150

ISBN : 978-2-86480-981-4

ISSN : 1633-5961

\section{Référence électronique}

Béatrice Fleury et Jacques Walter, «Promouvoir la diversité culturelle (2) ? », Questions de communication [En ligne], 14 | 2008, mis en ligne le 01 décembre 2008, consulté le 30 avril 2019. URL: http://journals.openedition.org/questionsdecommunication/877; DOI : 10.4000/ questionsdecommunication.877 


\title{
$>$ ÉCHANGES
}

\section{BÉATRICE FLEURY}

Université Nancy 2

Centre de recherche sur les médiations

Beatrice.Fleury@univ-nancy2.fr

JACQUES WALTER

Centre de recherche sur les médiations

Université Paul Verlaine-Metz

jacques.walter@univ-metz.fr

\section{PROMOUVOIR LA DIVERSITÉ CULTURELLE (2) ?}

\begin{abstract}
Résumé. - Deux contributions prennent la suite de celles publiées dans la $13^{\text {e }}$ livraison de Questions de communication et qui avaient pour objet la discussion des propositions de Joëlle Farchy et Jean Tardif. C'est à l'Asie émergente et à ses rapports avec l'Occident que l'économiste Louis Baeck s'intéresse, tandis que le sociologue, spécialiste des questions d'immigration, Ahmed Boubeker, envisage la globalisation au prisme de la ville et de l'ethnicité. Deux focales - l'une à large portée, l'autre plus restreinte - traitent donc d'un même phénomène et contribuent à aiguiser le regard et les connaissances sur la mondialisation et ses représentations.
\end{abstract}

Mots clés. - Mondialisation culturelle, économie, Asie, États-Unis, Europe, ville, ethnicité, frontière, identité, transculturalité, domination. 


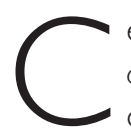

ette deuxième livraison des «Échanges » de la revue Questions de communication consacrés à la diversité culturelle se distingue de la précédente, déjà parce qu'aucun des deux contributeurs ne situe son propos dans le cadre d'un échange critique. En effet, contrairement aux chercheurs qui les ont précédés - Joël Augros (2008), Anne-Marie Autissier (2008), Philippe Bouquillion (2008), Tristan Mattelart (2008) -, Louis Baeck et Ahmed Boubeker ne tentent nullement une mise à l'épreuve des propositions théoriques de Joëlle Farchy (2008) et Jean Tardif (2008). En revanche, ils s'emploient à en développer un aspect, à large portée pour le premier qui traite de l'étonnante émergence des pays asiatiques dans l'économie mondiale et de ses effets, à portée plus restreinte pour le second qui s'intéresse à la recomposition ethnique des villes soumises au poids des vagues migratoires.

Fondées sur un ancrage différent, leurs contributions se rejoignent néanmoins, toutes deux articulant ce qui a trait au global et au local. En effet, si Louis Baeck part de considérations élargies sur l'économie mondiale, il en vient aux phénomènes locaux de réappropriation politique, économique, culturelle. Et si Ahmed Boubeker traite d'identités qui se créent et recréent sur des territoires définis, il en éclaire la spécificité par la prise en compte des mouvements migratoires, en lien aussi avec des phénomènes macro-économiques : « Les migrations nous révèlent ainsi que seul un appui sur des points d'ancrage permet de rendre compte de la construction du local à travers des dynamiques globales ».

Ce ne sont donc plus les médias ou l'industrie culturelle qui sont au fondement de l'analyse ici engagée, mais ce qui en structure le fonctionnement, les politiques et stratégies économiques pour l'un, le tissu social pour l'autre. Ainsi cette deuxième livraison de Questions de communication dédiée à un sujet largement débattu dans l'arène sociale conduit-elle à progresser dans la connaissance de phénomènes qui résultent de facteurs pluriels.

\section{Quand un économiste parle de mondialisation...}

Ce n'est pas sur le terrain du débat que Louis Baeck se situe. En revanche, il propose une étude à large portée sur la situation internationale en matière de mondialisation. Car ce Professeur émérite en économie, exerçant à l'Université catholique de Leuven, considère que « nous vivons à une époque charnière de la globalisation » où se conjuguent la montée en puissance de l'Asie et la crise financière de l'Occident. Pour en attester, il s'appuie notamment sur le rapport diffusé en 2007 par la 
Banque mondiale - Dancing with Giants - qui stipule que la Chine et l'Inde sont devenues les « nouvelles locomotives de l'économie mondiale ». Le vocable dont il use est à la mesure des transformations économiques et géopolitiques qui dessinent les contours du monde contemporain. Ainsi parle-t-il d'une « onde de choc » perceptible en différents secteurs, dont celui de la littérature centrée sur la mondialisation.

Ici, on est loin des considérations en usage dans les travaux que les chercheurs en sciences de l'information et de la communication, ou proches de la discipline, consacrent à la mondialisation culturelle. En revanche, comme le constataient les contributeurs de la précédente livraison des « Échanges », Louis Baeck considère que les changements ne sont pas seulement économiques et/ou géopolitiques, ils sont également d'ordre culturel. Mais, dans cette configuration, la culture est plus précisément entendue au sens des travaux que des spécialistes de différentes nationalités consacrent à ces questions. Louis Baeck s'attache donc à « illustrer la dialectique discursive en prise avec la mutation des rapports de force dans le domaine matériel ». Pour cela, il inscrit cette dialectique dans une large configuration où sont prises en compte tant l'influence de grands événements internationaux que celle des résistances identitaires. Autant de préalables qui s'avèrent indispensables pour comprendre les ressorts du monde contemporain.

Concernant les grands événements, Louis Baeck part d'un constat : la fin de l'Union soviétique puis l'ouverture de la Chine au capitalisme d'État ont pu être, un temps, interprétées comme des moments triomphants de l'économie de marché et donc du libéralisme à l'occidentale. Or, avec la récession des marchés occidentaux, l'heure n'est plus à l'optimisme mais à la remise en cause. D'autant que des résistances se font jour quant aux normes et modèles imposés par l'Occident, via la diffusion marchande et/ ou culturelle à l'échelle mondiale, via aussi certains coups de force (par exemple en Irak ou en Afghanistan). Avec cette conséquence : « Devant cette avalanche des forces armées dans la région riche en pétrole, les penseurs des cultures non occidentales estiment que mondialisation est un charmant néologisme qui dissimule le véritable projet, celui du contrôle géopolitique de la zone et de l'occidentalisation de la planète entière ».

Mais, Louis Baeck ne dresse pas seulement une cartographie de la situation contemporaine, il l'éclaire par un précédent historique qu'il considère comme étant précurseur de la mondialisation : I'Empire espagnol du XV| siècle, événement sur lequel la littérature contemporaine se serait montrée « notoirement aveugle ». Quels enseignements tire-t-il de cette mise en correspondance? Non seulement, cette époque vit le monde changer d'échelle - «L'Espagne se profila comme "centre" d'un Empire où, soidisant, le soleil ne se couchait jamais »-, mais elle vit aussi les modes de 
pensée se transformer. Ainsi l'Église fût-elle un acteur essentielle de cette évolution, elle qui, par le biais des « maîtres en scolastique de l'université de Salamanque », s'employa à théoriser la mondialisation. Mais si celle-ci décrivit et conceptualisa les mécanismes monétaires et la financiarisation de l'économie, il est un groupe, les arbitristes nationalistes (arbitristas), qui démontra les conséquences préjudiciables d'une mondialisation trop ambitieuse. Ainsi ces derniers firent-il le constat qu'un agrandissement d'échelle pouvait avoir pour conséquence d'entraîner « l'épuisement des forces vives », la crise des marchés, mais aussi celle des consciences.

Grâce à cette démonstration, on accède à une donnée essentielle de l'articulation entre économie, géopolitique et pensée. Qu'il s'agisse du choc de la mondialisation dont témoignent les écrits du XVle siècle, des effets engendrés par l'élargissement des frontières (avec à la clé les conquêtes coloniales au siècle suivant), la conscience de soi - et donc de l'autre - fait étrangement écho aux questions débattues aujourd'hui. Fort de cet exemple, et au vu de son expérience d'économiste, Louis Baeck décrit ensuite l'entrée puis l'expansion des pays émergents de l'Asie orientale dans l'ère industrielle contemporaine. II le fait en étudiant par le menu la structuration des marchés et les stratégies mises en œuvre, mais également en mettant à jour les conceptions économiques qui les accompagnent. Impressionnantes sont les procédures mises en place et les efforts engagés par ces pays émergents pour les soutenir. Si bien que la présentation que Louis Baeck fait ensuite de la situation américaine peut paraître inédite, habitués que nous sommes à envisager cette Nation sous l'angle de sa puissance. Et si l'on ajoute à ceci que nous sommes si exercés à fonder notre approche de la mondialisation sur les valeurs occidentales, nous ne pouvons que constater le manque d'acuité du regard que nous portons sur ces phénomènes. Une défaillance qui touche autant l'évaluation des ajustements auxquels procèdent les pays émergents par exemple de type politique - que les difficultés rencontrées aujourd'hui par certains pans de l'économie occidentale, dont américaine. Si, côté asiatique, les marchés connaissent un rattrapage accéléré qui prends corps dans une « culture de réseau », côté américain, les marchés s'affaiblissent, le budget de l'État est déficitaire, les « investissements étrangers [... . augmentent plus vite que l'investissement américain à l'étranger $\gg . .$. Comme dans les analyses de l'Empire espagnol du XVle siècle, des économistes partisans de conceptions opposées s'affrontent, certains défendant l'idée d'une « macro-économie sans frontières », d'autres plaidant pour « plus de modération et d'orthodoxie économique ». Ainsi les États-Unis connaissent-ils une mutation à différents niveaux dont l'une et non des moindres est d'ordre culturel, la candidature d'un Afro-américain - Barak Obama - à la présidentielle américaine de 2008 étant un « signal clair » de ceci.

Ce n'est donc pas sur le seul plan économique, ni sur celui des seules idées que Louis Baeck se positionne. C'est aussi sur une expérience 
affinée des cultures qu'il ancre sa réflexion. Ainsi précise-t-il qu'ayant été conduit à séjourner dans les pays asiatiques pour y donner cours et conférences, il a pu mesurer combien était importante la prise en compte du « tréfonds culturel comme un des déterminants du développement social et économique ». Plus précisément, il explique que, dans ses contacts « avec certains formateurs d'opinion de l'intelligentsia et avec des responsables politiques, [il a] senti que ces civilisations d'ancienne culture sont marquées d'une blessure ». L'Occident les ayant longtemps dominées, elles sont prêtes aujourd'hui à fournir maints efforts pour s'assurer «la reconquête de l'initiative historique ». Une disposition plus brûlante encore dans les pays musulmans que, de surcroît et de façon dommageable, les Occidentaux ont tendance à considérer comme un bloc monolithique. N'étant en mesure de comprendre ni les sentiments qui animent ces pays, ni le rôle du religieux - signe aussi de modernité -, les puissances occidentales, avec des disparités entre les États-Unis et l'Europe, cristallisent oppositions et rancœurs. Pour Louis Baeck, l'anthropologie culturelle et les philosophes postmodernistes sont à même de rectifier ce positionnement inappropriée, et donc d'ouvrir l'Occident à cet « autre » avec lequel il rencontre quelque difficulté à dialoguer. D'autant que l'Occident a beaucoup à apprendre de ces pays. II en est ainsi de l'Inde, à propos de laquelle l'économiste suggère : « Des concepts autres que les nôtres sur l'homme, sur son enracinement dans la société et sur les grands projets d'avenir devraient enrichir nos façons d'appréhender les références conceptuelles élargies pour la vie en société mondialisée. La globalisation a dynamisé et enrichi la mosaïque culturelle de notre planète $\gg$.

\section{Quand un sociologue de l'immigration parle de mondialisation...}

Comme en réponse à Louis Baeck, c'est à l'aune de la diversité culturelle qu'Ahmed Boubeker envisage la question de la globalisation. Se fondant sur des définitions proposées par Georges Balandier (1985:21), il évoque les traits saillants d'une modernité qui recompose des espaces, des groupes, des référents identitaires comme autant de «terres ignorées 》. D'emblée, comme le faisaient les contributeurs de la précédente livraison de Questions de communication, il souligne l'ambiguïté de la notion « qui sous-tend le clivage public entre une vision apocalyptique et une vision idyllique de la mondialisation ». À ce sujet, il dit le mérite de Joëlle Farchy et de Jean Tardif d'avoir su éviter le piège de la simplification à outrance pouvant conduire à faire perdre à des objets concrets leur lisibilité. Ainsi ne procède-t-il pas à une critique des contributions de ces derniers dont il dit d'ailleurs partager le point de vue -, mais à un prolongement 
de celles-ci via ses propres terrains de recherche. II envisage donc les questions soulevées à partir du thème de la ville et de l'ethnicité qu'il considère comme étant « des analyseurs de la globalisation ». Pourquoi ce choix? Parce que, selon lui, « lieu par excellence de la coexistence des cultures, la ville se présente comme un analgon du monde tandis que les mouvements migratoires sont eux-mêmes générés par l'inégalité des situations économiques et politiques à l'échelle de la planète ». Or, si l'on constate des ethno-stratifications dans la croissance urbaine, Ahmed Boubeker, à l'instar d'Arjun Appadurai (1998, 2006), y voit la manifestation d'un régime d'incertitudes de la vie sociale dont la réaction de repli est un aspect. Aussi diversité et crise sont-ils deux aspects à mettre en correspondance, de même que le sont interculturalité et « défi politique d'un nouvel être ensemble ». Une articulation à laquelle s'emploie le sociologue, en travaillant sur «le phénomène urbain comme mode de vie [qui] s'impose comme le site même de l'interculturalité dont les seuils sont à la fois matériels et symboliques, physiques et rationnels ».

Le sociologue prévient: dans le cadre urbain, on n'a pas affaire à une culture partagée et on est loin du «mythe d'une convivialité culturelle », mais plutôt, si l'on suit Ulf Hannerz (1980), à « des modes d'entente, de croisement, de contact qui usent des ressources des civilités sans requérir de réciprocité de perspectives $\gg$. Par la suite, on voit l'espace commun se distribuer « en différents espaces qui s'emboîtent les uns les autres en référence à des mondes distincts ». Chevauchement, superposition, enchâssement, bricolage, appropriation « tracent [donc] les frontières du territoire et la topographie de la mémoire ». Ce qui conduit à solliciter une conception plurielle de la notion de frontière, cette dernière ayant pour particularité de ne pas seulement se définir selon des caractéristiques physique, mais d'être totalement en prise avec une dimension symbolique à partir de laquelle se négocient des identités.

C'est un monde mouvant, situé au carrefour entre mobilité et sédentarité que décrit Ahmed Boubeker. Agi par la circulation des hommes, des marchandises ou des informations, il « fait richesse et productivité sociale de sa capacité à agencer des activités et des groupes [...], à jouer sur des hybridations et des rencontres ». Ainsi la transculturalité est-elle au cœur de la démarche du sociologue, soucieux de comprendre comment se constituent et se construisent des espaces urbains, envisagés comme « un grand laboratoire [...] de la ville monde ». D'où le recours à une analyse qui met à la fois en évidence ce qui a trait au mouvement (processus, dynamiques, relations) et ce qui relève de l'inscription dans un territoire (foyer, carte, frontière). L'une de ses assertions se rapportant à la culture va dans ce sens : «Au-delà de toute vision substantialiste, la culture s'affirme comme une dimension des phénomènes sociaux relevant de différences situées. Elle sert de rappel, non pas simplement sur un registre de la 
nostalgie des origines, mais pour préparer une mobilisation des identités de groupe, pour élargir une capacité d'action sur le monde et sur soi, pour faire de son vécu un moyen de pouvoir vivre d'autres vies ». D'où la question du politique à laquelle Ahmed Boubeker en vient naturellement, un domaine auquel il assigne, dans le cadre de son raisonnement, la mission de trouver « la bonne formule permettant de gérer la diversité sans éclatement ».

\section{Conclusion}

Si Louis Baeck et Ahmed Boubeker évoquent la question de la suprématie du modèle occidental, chacun accorde à celle-ci une place et un sens distincts dans le monde contemporain. Pour Louis Baeck, cette suprématie est en crise. Ce qui est manifeste à la fois sur le plan économique avec l'effet de rattrapage que connaissent aujourd'hui les pays émergents, et sur le plan politique avec les stratégies de résistance mises en œuvre par certains groupes aux normes et valeurs imposées par le monde occidental. Ainsi voit-on se profiler une situation charnière montrant la faillite d'un système qui s'est longtemps pensé comme la norme absolue. Pour Ahmed Boubeker, la suprématie - ou domination - se conjugue surtout sur le mode américain et est envisagée selon son caractère inaccessible «pour la grande majorité des nations du monde ». Dans un cas, la diversité existe de fait, mais les démocraties occidentales ne sachant pas la voir ou la comprendre, elles encourent le risque de se voir distancées. Dans l'autre, elle est une forme de combat en vu de préserver la liberté inconditionnelle de l'homme dans la cité. L'économiste envisage donc plutôt la suprématie sous l'angle de l'effet boomerang qu'elle peut engendrer, le sociologue de l'immigration sous l'angle de la quête identitaire.

En deux livraisons, Questions de communication est loin d'avoir épuisé l'ensemble des problèmes touchant à la diversité culturelle. Mais en sollicitant des chercheurs d'origine disciplinaire différente, la revue s'est employée, autant que faire se peut, à démontrer que la compréhension de ces questions ne peut passer que par des approches heuristiques diverses et, par là, complémentaires.

\section{Références}

Augros J., 2008, « Glocalisation, runaway et local production. Deux ou trois choses que je sais d'elles », Questions de communication, 13, pp. 225-238.

Appadurai A., 1998, Après le colonialisme. Les conséquences culturelles de la globalisation, trad. de l'anglais par Fr. Bouillot, Paris, Payot, 2001. 
- 2006, Géographie de la colère. La violence à l'âge de la globalisation, trad. de l'anglais par Fr. Bouillot, Paris, Payot, 2007.

Autissier A.-M., 2008, « Pour une réorientation fondamentale des politiques culturelles », Questions de communication, I3, pp. 239-250.

Balandier G., 1985, Le détour. Pouvoir et modernité, Paris, Fayard.

Bouquillon Ph., 2008, «La diversité culturelle. Une approche communicationnelle», Questions de communication, 13, pp. 25I-268.

Farchy J., 2008, « Promouvoir la diversité culturelle. Les limites des formes actuelles de régulation », Questions de communication, 13, pp. 17| - 195.

Hannerz U., 1980, Explorer la ville. Éléments d'anthropologie urbaine, trad. de l'anglais par I. Joseph Paris, Éd. de Minuit, 1983.

Mattelart Tr., 2008, « Pour une critique des théories de la mondialisation culturelle », Questions de communication, 13, pp. 269-287.

Tardif J., 2008, « Mondialisation et culture : un nouvel écosystème symbolique », Questions de communication, 13, pp. 197-223. 